\title{
Design Matters: Mid-Term Results from a Multi-Design Fuel Economy Feedback Experiment
}

\author{
Tai Stillwater ${ }^{*}$ and Kenneth S. Kurani \\ UC Davis PH\&EV Center, University of California, Davis, \\ 1590 Tilia Street, Davis, CA 95616 \\ tstillwater@ucdavis.edu
}

\begin{abstract}
Energy feedback to drivers is one method to engage drivers in energy saving driving styles. In contrast to the occasional broadcasting of general driving tips, in-vehicle energy feedback gives drivers access to accurate information about their specific driving situation on an ongoing basis. The increasing prevalence of such feedback in new vehicles suggests a belief that ongoing, in-vehicle feedback is better. However, there is little reliable evidence of the effectiveness of energy feedback in real-word driving in passenger vehicles. This study begins to fill this gap. Participants are given a commercially-available fuel consumption display and recording device to use in their personal vehicle for two months. For the first month the display is blank as the device records a baseline of driving and fuel consumption. For the second month the display is switched on to show drivers one of three feedback designs. This paper presents preliminary results $(\mathrm{N}=75)$ of a larger study that will include 150 drivers along the California-Nevada Interstate- 80 corridor. Using a mixed-effects linear model, we find an average driving efficiency improvement of between $1.5 \%$ and $6 \%$ (gallons/100 miles) between the without- and withfeedback months, depending on the feedback designs. Categorizing trips into types based on distance and multiple speed characteristics, there are differences in the apparent effectiveness of feedback across trip types. Finally, an overall decrease in fuel consumption of $10 \%$ between periods was observed. While approximately $3 \%$ of that is explained by changes in driving behavior, the remaining $7 \%$ is due to reduced VMT.
\end{abstract}

Keywords: Driver Behavior, HMI, Human Machine Interface, Behavior Change, Energy Conservation, Ecodriving.

\section{Introduction}

Past research indicates the influence drivers can have on passenger vehicle fuel economy (1-3). A suite of energy-saving behaviors has come to be known as ecodriving-including moderating top speeds and acceleration and increased coasting (especially approaching stops). However, the potential improvements from ecodriving are mediated or structured by roadway design, traffic levels, competing norms about driving styles, and drivers' own interest and knowledge regarding eco-driving.

\footnotetext{
* Corresponding author.
} 
In this paper, we focus on the impact of in-vehicle fuel economy feedback on fuel consumption, using a framework of driver attitudes, interest, and knowledge to help explain driver behavior. To make precise measures of the effectiveness of feedback, we test the effectiveness of three common feedback styles on ecodriving behaviors on-road fuel consumption using a large sample size and two months of recorded driving per observation. The experiment will eventually reach a sample size of 150 drivers in cities and towns along the I-80 corridor from San Francisco, CA to Reno, $\mathrm{NV}$, for a projected total of 25 driver-years by the end of the project (of which only $1 / 4$ has been completed to date). Here, we present preliminary results based on the first 36 drivers from Davis, CA, representing six driver-years of study.

\section{Description of the Experiment}

This study extends the current body of knowledge of ecodriving feedback efficacy by testing multiple versions of fuel economy feedback in a two month natural driving experiment. To make the best possible estimate of the efficacy of the three tested designs, thirty to forty participants will be enrolled in each four distinct regions in two states, for a total of 150 individual drivers and 25 vehicle years in the experiment (12.5 baseline vehicle-years and 12.5 treatment vehicle-years).

\subsection{Study Regions and Household Selection Process}

To ensure that the estimates of fuel savings can be generalized many driving situations, four distinct metropolitan regions along the I-80 corridor were selected for study, comprising San Francisco, Davis, and Sacramento, CA as well as Reno NV. This paper details the preliminary results from Davis, CA.

A household sample was selected with the participation of the North American American Automobile Association (AAA). The recruitment criteria included ownership of at least one non-hybrid post-1996 model year vehicle, AAA insurance of $\$ 100,000$ in accident coverage, $\$ 300,000$ per occurrence, and $\$ 50,000$ in property damage. A letter stating the general outline of the study was sent to a sample of 3-500 qualifying drivers in each region. The letter included a link to a recruiting survey which would enter the participant into our pool of possible participants. Participants and interested co-insurees were then enrolled in the study.

Each participating household was given a display to use for one month without receiving feedback to record a baseline driving period. After one month a researcher would return to the household to reprogram the device to enable the fuel economy feedback feature. The household would then use the updated device and view energy feedback for the following month, until a researcher would return to the household a final time to interview the participants about their experience using the display as well as to uninstall the display from the household vehicle and retrieve the driving data.

\subsection{Screen Selections}

Three feedback display designs were selected that span the range of designs tested for user comprehension and satisfaction in the 2010 NHTSA Fuel Economy Driver Interface Report (4). 
Table 1. Feedback Designs used in the Experiment

\begin{tabular}{cl}
$\begin{array}{c}\text { Screen } \\
\text { Name }\end{array}$ & \multicolumn{1}{c}{ Description } \\
\hline Numbers & $\begin{array}{l}\text { Real-time MPG (1) , trip average } \\
\text { MPG (2), current value shown by a } \\
\text { green bar chart(A) the mean value is } \\
\text { set to the EPA combined cycle fuel } \\
\text { economy rating for that vehicle (B) } \\
\text { the current value is also shown in } \\
\text { numeric form (C). }\end{array}$ \\
Accelerator & $\begin{array}{l}\text { Instantaneous acceleration bar (2) and } \\
\text { trip-level leaf representation of fuel } \\
\text { economy (1) where the center point } \\
\text { (A) represents the EPA combined } \\
\text { cycle Fuel Economy Rating. }\end{array}$ \\
Shrubbery & $\begin{array}{l}\text { Short term (1A) and trip-level (2) leaf } \\
\text { representations of fuel economy. The } \\
\text { mean value of the bars is set to the } \\
\text { EPA combined cycle fuel economy } \\
\text { rating for that vehicle (B). }\end{array}$
\end{tabular}

The implications for the design and sample size of the field test are discussed below. The selection of three screens from the NHTSA report's seven representative screens is based on three factors: reducing cognitive load by reducing the number of different screen types we will have any of our drivers view (measured by user response time), improving comprehension (measured by a user task with a binary correct/incorrect result), and increasing user satisfaction (measured by user selfreports). The three screens are implemented nearly as shown in the NHTSA report, although higher-contrast colors are used to increase visibility in the vehicle.

\subsection{Data Recording Details}

To estimate the influence of the three feedback designs on fuel consumption as closely as possible, detailed data were recorded from each vehicle. The study used the DrewTech DashDaq display and data logger to retrofit participant's own vehicles with a graphical feedback display. Fuel-consumption related data were recorded from the On Board Diagnostic (OBD-II) port at an average of $20 \mathrm{hz}$ (twenty records per second) for the duration of each recorded trip. Standard conversions were used to calculate 
fuel consumption from recorded MAF (Mass Air Flow) sensor readings, and distance traveled from the VSS (Vehicle Speed Sensor). For vehicles using the speed-density engine control strategy, which do not include a MAF sensor, synthetic MAF values were calculated in the DashDaq using the vehicle engine displacement, intake air temperature, engine RPM, and an assumption of $80 \%$ volumetric efficiency. When available, the Lambda sensor was also recorded to adjust the fuel consumption values in periods of rich or lean fuel-air mixtures. In addition to the OBD-II engine data, a GPS receiver attached to the DashDaq was used to log altitude at a $1 \mathrm{hz}$ rate for estimates of elevation and road grade.

To enable proper estimation of the effect of the interface in vehicles with multiple drivers, the display was programmed to allow each driver to enter a personal driver identification number, allowing up to three drivers per vehicle to be recorded.

\section{Data Treatment and Analysis}

The summary data for each trip was collected in a single $\mathrm{R}$ data set for analysis. First, the trips were clustered into distinct types as described in the Trip Type section below to test for differences in effectiveness of the feedback based on the driving pattern (e.g. city and highway trips). The data analysis then proceeded in order of model complexity to determine the true effect of the devices. Fuel consumption and distance were then used to calculate an overall GP100M (Gallons per 100 Miles) fuel consumption measure for each trip.

An overall T-test was used to make an estimate of un-adjusted driving data (wherein the fuel consumption was not controlled for factors such as the vehicle or driver, temperatures, or trip types). Then a T-test for each feedback design was performed to make an unadjusted estimate for each design.

To adjust for changing drivers, vehicles, temperatures, and driving patterns a mixed effects linear regression model was fit to the data. The regression model includes a consumption offset for each driver-vehicle combination to account for the different intrinsic efficiency of various vehicles. The model then includes multiple explanatory factors besides the experimental treatment to disentangle changes in efficiency due to temperature changes, changes in trip patterns, and changes in driver or vehicle during the course of the experiment. Two regression models were fit to the data, the first to test the average effect of the feedback on different driving patterns, and the second regression tested the differential effect of the three feedback designs. The models and interpreted output are shown in Tables 4 and 5 and Figures 1 and 2.

\section{Trip Types}

To reduce drive-cycle variance in the model, and to assess the effectiveness of feedback in varying drive-cycles, trips were clustered using the K-means methodology to identify trips that have distinct drive-cycle characteristics. The four dimensions used for clustering are the trip distance, mean speed, maximum speed, and number of stops. 


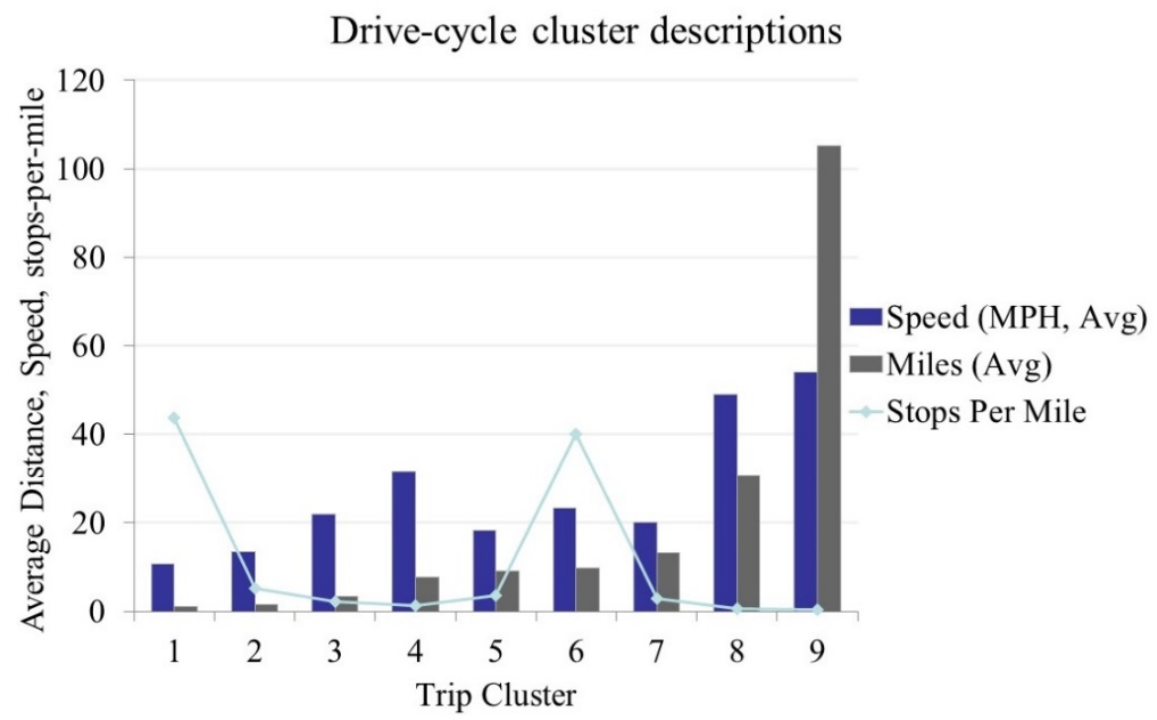

Fig. 1. Trip Types

\section{$4 \quad$ Results}

The overall t-test between experimental periods shows a statistically significant decrease in fuel consumption per mile of $10 \%(p=0.007)$, a value that generally fits within previous estimates. However, this effect is not primarily due to driving behavior. Only 3\% (on average) decrease in fuel consumption is due to driving behavior as given by the model methodology. The remaining $7 \%$ decrease is due to reduced driving. A further analysis with the final dataset will hopefully clarify if those changes were seen by chance, or if they are an effect of the interfaces as well.

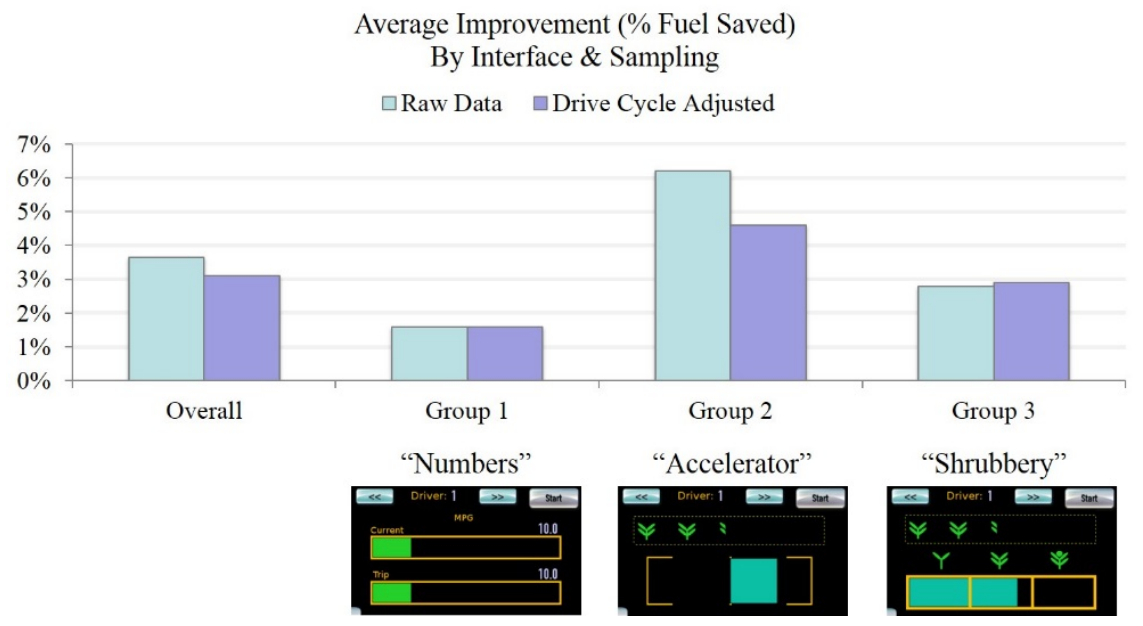

Fig. 2. Trip Type Model Testing Effectiveness of Feedback in Varying Driving Circumstances 
To provide realistic estimates that control for vehicle differences, driver, drivecycle and temperature, a mixed-effects linear model was used to estimate the average changes in on-road fuel consumption by each driver due to the feedback. The model results are shown below in graphical format.

The trip model (Table 4 and Figure 1) dependent variable is GP100M (Gallons/ 100 Miles driven). Trips below 0.25 miles are excluded, N=6103 Trips, 47 vehicledriver combinations, $\mathrm{R} 2=0.49$. Trip type 1 (she category with the shortest trips) is used as the model baseline. Explanatory variables include temperature difference from $72 \mathrm{~F}$ (to account for heating as well as cooling), average road grade, and experimental phase*trip type interaction term. Unique driver-vehicle combinations are included as the random-effect grouping to control for differences in average fuel consumption between vehicle types.

The driving behavior model shows changes of $1.75 \%(p=0.006)$ for the Numbers display, 6\% (p=.018) for the Accelerator display, and 3\% (p=0.3) for the Shrubbery display (a net increase in consumption).

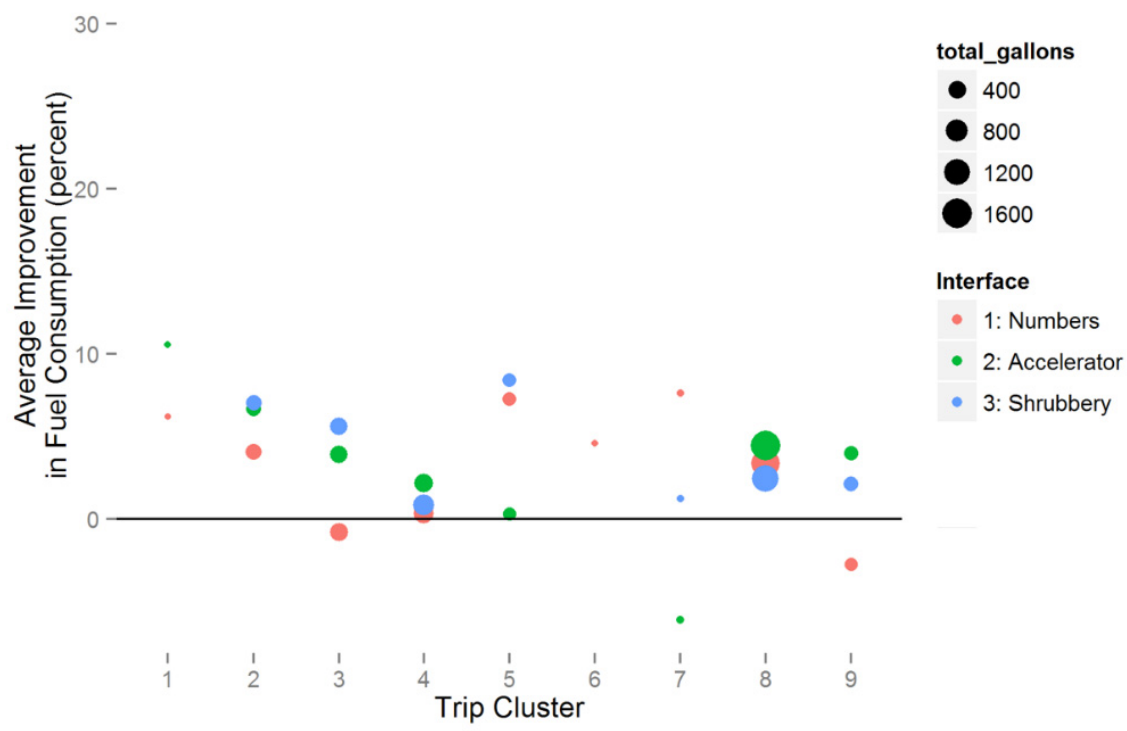

Fig. 3. Average Fuel Consumption and Reduction in Fuel Consumption across Trip Categories. Results are shown for a trip with $72^{\circ}$ ambient temperature, $0 \%$ grade.

The Trip Model shows that there is a significant decrease in fuel consumption in almost all trip types and interface designs. A number of trends in this analysis are worth noting for future design studies: the most effective design changes between shorter trips (1-4, the shrubbery display was the most useful) and longer trips (8-9, where the accelerator display was the most useful). This suggests that different information is more valuable during different driving periods. 


\section{Conclusions}

The ecodriving experiment conducted here examines 1) the efficacy of in-vehicle feedback to drivers on energy consumption outcomes, and 2) the differential efficacy of three different designs of such feedback, i.e., what information is provided through what graphical representation. Results from the first set of drivers indicate that energy consumption is reduced in the presence of feedback. Controlling for contextual differences between the without and with feedback phases, the presence of three feedback designs are correlated with reductions in fuel consumption of $8 \%, 4 \%$ and $2 \%$, respectively, for a median trip of the Short Arterial type. The type of driving appears also to be important; feedback had little or no impact on energy consumption for long-distance highway driving (Regional type). All other trips showed reduction in energy consumption of between 4 and $6 \%$.

A mixed-effects linear model was found to be better than a direct t-test of fuel consumption because it controls properly for vehicle effects and trip factors (e.g., drive-cycle and ambient temperature), making it possible to separate changes in trip patterns from changes in ecodriving. However, for large sample tests, a more flexible mixed effects model should be used to fully account for changes in trip patterns that could also be the result of ecodriving feedback.

\section{$6 \quad$ Next Steps}

This paper presented the preliminary data from a group of 36 drivers from a future total of 150 drivers in a wider variety of driving contexts. This analysis will be extended to incorporate the final data set in early 2013.

In addition, the survey results and interviews from the participating drivers will be incorporated to help determine why driver feedback was or was not effective, as well as to help distinguish why some feedback styles are more useful than others.

Acknowledgement. Funding for this project came from the Oak Ridge National laboratory in conjunction with the US Department of Energy.

\section{References}

1. Greene, D.L.: Driver Energy Conservation Awareness Training: Review and Recommendations for a National Program, Oak Ridge National Laboratory (1986)

2. Ando, R., Nishihori, Y., Ochi, D.: Development of a System to Promote Eco-Driving and Safe-Driving. In: Balandin, S., Dunaytsev, R., Koucheryavy, Y. (eds.) ruSMART 2010. LNCS, vol. 6294, pp. 207-218. Springer, Heidelberg (2010)

3. Barkenbus, J.N.: Eco-driving: An overlooked climate change initiative. Energy Policy 38(2), 762-769 (2010)

4. Jenness, J.W., Singer, J., Walrath, J., Lubar, E.: Fuel Economy Driver Interfaces: Design Range and Driver Opinions Report on Task 1 and Task 2 (2009) 